\section{Qualidade de Vida Relacionada à Saúde de Pacientes Oncológicos sob Cuidados Paliativos}

\author{
Health Quality of Life Related to Cancer Patients' Health \\ Under Palliative Care
}

\section{RESUMO}

O texto discute os principais resultados obtidos na dissertação do Mestrado em Psicologia da Saúde pela Universidade Católica Dom Bosco (UCDB) concluído em 2012, que teve por objetivo avaliar a Qualidade de Vida Relacionada à Saúde (QVRS) de pacientes oncológicos sob cuidados paliativos. Trata-se de um estudo quantitativo, descritivo e de corte transversal, aprovado pelo Comitê de Ética em Pesquisa da UCDB, de Campo Grande, Mato Grosso do Sul. A amostra compôs-se de 70 participantes que realizavam acompanhamento paliativo no Hospital do Câncer de Dourados/MS. Foram aplicados dois instrumentos para coleta de dados: o Questionário Sociodemográfico Ocupacional e um instrumento específico para avaliar a QVRS em oncologia, o EORTC $Q L Q-C_{3}$. Os resultados demonstraram que a identificação objetiva dos domínios, quando correlacionados às variáveis sociodemográficas ocupacionais, pode favorecer o direcionamento das intervenções assistenciais que a equipe multidisciplinar oferece ao paciente oncológico em relação ao enfrentamento da doença e aos efeitos adversos do tratamento, o qual, ocasionalmente, pode comprometer sua QVRS.

Palavras-chave: Qualidade de Vida Relacionada à Saúde. Oncologia. Cuidados Paliativos.

\section{ABSTRACT}

This paperwork discusses the main results of the Health Psychology Master Degree Dissertation of Universidade Católica Dom Bosco (UCDB) concluded in 2012, which aimed to assess the health quality of life of cancer patients under palliative care. This is a quantitative, descriptive and cross-sectional study, approved by the Ethics Research Department of (UCDB) in Campo Grande, Mato Grosso do Sul. The sample consisted of 70 participants who underwent palliative monitoring care at the Cancer Hospital in Dourados/MS. Two instruments were applied to collect data: a Social Demographic
Cintia Rachel Gomes

\section{SALES}

Centro Universitário da Grande Dourados. Mato Grosso do Sul, Brasil

\section{José CARLOS Rosa}

Pires

Universidade Católica Dom Bosco. Mato Grosso do Sul, Brasil

\section{IDALINA CRISTINA} Ferrari Júlio

Universidade Estadual de Mato Grosso do Sul. Mato Grosso do Sul, Brasil 
Occupational Questionnaire and a specific instrument to assess the health quality of life in Oncology the EORTC QLQ - C 30 . The results showed that a target identification of domains, when related to Social Demographic Occupational variability, may favor the assistance interventions that the multidisciplinary team offers to cope with the disease and side effects of cancer treatment in order to improve those individuals health quality of life.

Keywords: Quality of life. Oncology. Palliative Care.

\section{INTRODUÇÃO}

O câncer é definido como uma doença crônico-degenerativa e, por isso, é considerado como um problema de saúde pública. O diagnóstico precoce contribui de forma significativa para a reabilitação física, social e psicológica do doente [4]. Porém, seu diagnóstico confirmado abrange, de forma gradativa e simultânea, aspectos físicos, psicossociais, emocionais e financeiros do paciente. Por muitos anos, o tratamento do câncer estava vinculado ao ponto de vista da morbidade, mortalidade ou cura. No entanto, os avanços da medicina já permitem o controle dos sintomas e estadiamento de tal doença.

De acordo com as implicações psicológicas, tanto na etiologia quanto na terapêutica do câncer, um crescente interesse em estudos que avaliem a QV, tem buscado demonstrar a autopercepção de bem ou mal-estar e sua contribuição na evolução biológica da enfermidade, podendo constituir-se como um cofator terapêutico. Assim, a avaliação da $\mathrm{QV}$ poderia promover eficácia e segurança aos tratamentos propostos [5].

No entanto, a Qualidade de Vida (QV) é um conceito que, nas últimas décadas, tem sido alvo crescente de interesse tanto na literatura científica, como entre o público em geral. Na literatura específica, observa-se uma diversidade de significados para o termo $\mathrm{QV}$, com variadas abordagens teóricas e inúmeros métodos para a medida do conceito. Instrumentos que mensurem a QV têm sido desenvolvidos e avaliados, uma vez que permitem a identificação dos problemas em áreas relativas ao estado emocional e estado físico geral, bem como a interação social e o delineamento de programas adequados de intervenção, possibilitando um acompanhamento multidisciplinar ao paciente oncológico [3].

Recentemente, o conceito de Qualidade de Vida Relacionado à Saúde (QVRS) passou a ser considerado de extrema importância, pois revelou que a eficácia do tratamento não pode ser determinada simplesmente pela perspectiva do tempo livre de doença ou de sobrevida. Partindo dessa premissa, a pesquisa buscou avaliar a QVRS dos pacientes oncológicos sob cuidados paliativos cadastrados no Centro Ambulatorial de Tratamento de Câncer, no município de Dourados, Mato Grosso do Sul, em 2011. Além da interface com a pesquisa, esse trabalho também visa o envolvimento multiprofissional, a fim de fomentar discussões e o aprimoramento profissional em relação à assistência ao paciente oncológico.

Diversos estudos têm proposto, a partir de delineamentos qualitativos e quantitativos (esse último, por meio de instrumentos genéricos e específicos), identificarem, mensurarem e avaliarem domínios afetados pela doença no indivíduo com câncer. Considera-se um desafio a aplicação clínica frente aos domínios propostos a serem avaliados e 
mensurados, pois compreende-se os inúmeros efeitos colaterais e secundários advindos do câncer e de seu tratamento, como alterações estéticas (mutilações), sociais, funcionais e de comportamento, os quais incidem de forma peculiar em cada sujeito.

Mesmo com o uso crescente no âmbito nacional e internacional de escalas de avaliação de QV, não há no Brasil uma medida de avaliação desse constructo voltada especificamente para a população em cuidados paliativos. Por isso, teve início a tradução, a adaptação cultural e a validação no Brasil do instrumento Palliative Outcome Scale (POS), que apresenta validade convergente satisfatória quando comparado às dimensões do EORTC QLQC-30 [2].

No entanto, a maioria dos instrumentos $(94,4 \%)$ busca avaliar a QV do paciente de forma multidimensional, incluindo aspectos físicos, emocionais, sociais, econômicos, espirituais, além do relacionamento com a família e com a equipe de saúde. Entende-se que essa tendência seja decorrente da compreensão de que as necessidades dos pacientes oncológicos sob cuidados paliativos devem ser identificadas de forma absoluta, a fim de implementar medidas pautadas no alívio do sofrimento e na melhora da QV [2].

\section{MATERIAIS E MÉTODO}

Tipo de Estudo

Trata-se de um estudo de caráter quantitativo, descritivo e de corte transversal, aprovado pelo Comitê de Ética em Pesquisa da Universidade Católica Dom Bosco (CEP/ UCDB), processo no oo8/11, de Campo Grande, Mato Grosso do Sul.

\section{Local do Estudo}

O estudo foi realizado no Hospital do Câncer, no município de Dourados, Mato Grosso do Sul.

\section{Coleta de Dados}

Para a coleta de dados foi utilizado um questionário de identificação, construído para a coleta de dados sociodemográficos (idade, escolaridade, sexo, cor, estado civil, religião, atividade laboral, renda mensal e familiar, prática de atividades físicas) e dos dados clínicos e terapêuticos (quantidade de internações após a confirmação do diagnóstico oncológico, tipo de tratamento - quimioterapia e/ou radioterapia - duração do tratamento e diagnóstico clínico). Para a avaliação da QVRS, utilizou-se o instrumento European Organization for Research and Treatment of Cancer Quality of Life Questionnaire - Core 30 (EORTC QLQ C-30), considerado um questionário de Qualidade de Vida Relacionado à Saúde (QVRS) devidamente validado para a população brasileira para uso específico em pacientes com câncer [1]. 
Após a coleta sistemática em 70 participantes, os dados foram inseridos em planilhas do software Excel e, em seguida, deu-se a análise estatística. Para a caracterização da amostra, foi realizada uma análise descritiva dos resultados, com frequência absoluta e relativa. A caracterização da amostra foi comparada com os domínios do instrumento EORTC QLQC-3o por meio do teste não paramétrico da Mediana de Mood. Esse teste é indicado quando se compara grupos pequenos ou quando há ausência de distribuição normal de probabilidade, pois altera minimamente os resultados atípicos observados. O teste da Mediana de Mood foi utilizado nessa amostra devido a disparidade apresentada em todos os domínios, isto é, distribuição anormal de probabilidade. O programa utilizado foi Minitab na versão 14.0 for Windows. O nível de significância foi de $5 \%$ ( $\mathrm{p} \leq \mathrm{0.05}$ ), ou seja, todos aplicados com $95 \%$ de confiabilidade.

\section{RESULTADOS}

A amostra inicial, com 105 participantes, foi selecionada de forma conveniente, mediante critérios de inclusão. Porém, cinco dos indivíduos selecionados participaram apenas do projeto piloto, a fim de verificar a viabilidade, adaptação e o tempo de duração da pesquisa. Apenas 70 entrevistas foram concluídas, devido a 30 dos pacientes selecionados faltarem ou irem a óbito. A coleta foi obtida entre os meses de junho e julho de 2011, mediante agendamento hospitalar de retorno ou consulta. A abordagem se deu antes ou após a realização do atendimento terapêutico (quimioterapia ou radioterapia) ou da consulta médica. No entanto, a amostra se caracterizou de forma homogênea, uma vez que todos os pacientes estavam em seguimento ambulatorial de tratamento paliativo.

Em relação ao perfil das variáveis sociodemográficas ocupacionais e terapêuticas (Tabela 1$)$, evidenciamos o predomínio do sexo masculino $(65,71 \%)$ com a prevalência do câncer de próstata. A maior frequência foi de 60 anos (60\%), cor predominante branca $(65,71 \%)$, católicos $(81,43 \%)$, casados $(68,57 \%)$, ensino fundamental incompleto $(77,14 \%)$, não exercem atividades laborais $(81,16 \%)$, renda familiar bruta com mais de um salário mínimo (76,81\%).

Percebeu-se que os participantes da pesquisa eram advindos de diferentes estratos sociais e a renda familiar bruta era, em geral, de pouco mais de dois salários mínimos, o que pode dificultar o acesso à escolaridade e, consequentemente, à adesão de informações pertinentes à prevenção e ao tratamento adequado do câncer. Também, nota-se que, a maioria possui um vínculo religioso, podendo ser o principal responsável em motivá-los a continuar o acompanhamento e o tratamento clínico.

A maioria dos participantes não praticava alguma atividade física $(65,71 \%)$ e sua terapêutica não ultrapassou cinco anos (60\%). Foram submetidos a dois tipos de tratamentos oncológicos - quimioterapia e radioterapia - $(60,29 \%) \mathrm{du}$ rante o curso da doença, contudo, tiveram apenas uma internação (55,07\%). Outro fato que merece destaque é a procedência da população em estudo, embora 55,38\% do total fossem de Dourados/MS, os demais participantes eram de regiões próximas, e a 
maioria dependia de transporte fornecido pela prefeitura de sua cidade de origem, o que demandava tempo de espera e de deslocamento. Além disso, observou-se que o atendimento é realizado por ordem de chegada e o tempo de espera no ambulatório para consulta ou tratamento específico é, na maioria das vezes, demorado.

Na Tabela 2 apresentamos o percentual de diagnósticos dos pacientes participantes do estudo, com predomínio do sexo masculino $(65,71 \%)$ com câncer de próstata (37,14\%) e câncer de esôfago (12,86\%), seguido do câncer de mama no sexo feminino $(\mathbf{2 1}, \mathbf{4 3} \%)$. Devido à disparidade no restante dos diagnósticos, não houve valor estatisticamente significativo a ser discutido.

Tabela 1 - Perfil das variáveis sociodemográficas ocupacionais e terapêuticas dos pacientes atendidos no Hospital do Câncer - Dourados/MS, 2011.

\begin{tabular}{|c|c|c|c|}
\hline VARIÁVEIS & & $\mathbf{N}$ & $\%$ \\
\hline \multirow{2}{*}{ Sexo } & Feminino & 24 & $34,29 \%$ \\
\hline & Masculino & 46 & $65,71 \%$ \\
\hline \multirow{2}{*}{ Escolaridade } & Até Fundamental incompleto & 54 & $77,14 \%$ \\
\hline & Fundamental ou mais & 16 & $22,86 \%$ \\
\hline \multirow{2}{*}{ Religião } & Católico & 57 & $81,43 \%$ \\
\hline & Outros & 13 & $18,57 \%$ \\
\hline \multirow{2}{*}{ Praticante da Religião } & Não & 10 & $14,71 \%$ \\
\hline & Sim & 58 & $85,29 \%$ \\
\hline \multirow{2}{*}{ Cor } & Branca & 46 & $65,71 \%$ \\
\hline & Outras & 24 & $34,29 \%$ \\
\hline \multirow{2}{*}{ Estado Civil } & Casado & 48 & $68,57 \%$ \\
\hline & Outros & 22 & $31,43 \%$ \\
\hline \multirow{2}{*}{ Trabalha } & Não & 56 & $81,16 \%$ \\
\hline & $\operatorname{Sim}$ & 13 & $18,84 \%$ \\
\hline \multirow{2}{*}{ Aposentado } & Não & 33 & $47,14 \%$ \\
\hline & Sim & 37 & $52,86 \%$ \\
\hline \multirow{2}{*}{ Afastado } & Não & 14 & $42,42 \%$ \\
\hline & $\operatorname{Sim}$ & 19 & $57,58 \%$ \\
\hline \multirow{2}{*}{ Atividade Física } & Não & 46 & $65,71 \%$ \\
\hline & Sim & 24 & $34,29 \%$ \\
\hline \multirow{2}{*}{ Terapêutica } & Até cinco anos & 42 & $60,00 \%$ \\
\hline & Acima de cinco anos & 28 & $40,00 \%$ \\
\hline \multirow{2}{*}{ Tratamento } & Dois & 41 & $60,29 \%$ \\
\hline & $\mathrm{Um}$ & 27 & $39,71 \%$ \\
\hline \multirow{2}{*}{ Idade } & Até 60 anos & 28 & $40,00 \%$ \\
\hline & Mais que 60 anos & 42 & $60,00 \%$ \\
\hline
\end{tabular}




\begin{tabular}{lccc}
\hline \multirow{2}{*}{ Salário } & Até um s.m. & 50 & $73,53 \%$ \\
\cline { 2 - 4 } & Mais que um s.m. & 18 & $26,47 \%$ \\
\hline \multirow{2}{*}{ Renda Familiar Bruta } & Até um s.m. & 16 & $23,19 \%$ \\
\cline { 2 - 4 } & Mais que um s.m. & 53 & $76,81 \%$ \\
\hline Internações & Até uma & 38 & $55,07 \%$ \\
& Mais que uma & 31 & $44,93 \%$ \\
\hline
\end{tabular}

Tabela 2 - Percentual de diagnósticos dos pacientes atendidos no Hospital do Câncer Dourados/MS, 2011.

\begin{tabular}{lll}
\hline DIAGNÓSTICO & N & $\%$ \\
\hline Próstata & 26 & $37,14 \%$ \\
\hline Mama & 15 & $21,43 \%$ \\
\hline Esôfago & 9 & $12,86 \%$ \\
\hline Pulmão & 4 & $5,71 \%$ \\
\hline Intestino & 2 & $2,86 \%$ \\
\hline Mieloma múltiplo & 2 & $2,86 \%$ \\
\hline Colón & 2 & $2,86 \%$ \\
\hline Coluna & 2 & $2,86 \%$ \\
\hline Estômago & 1 & $1,43 \%$ \\
\hline Glândula parótida & 1 & $1,43 \%$ \\
\hline Sarcoma de partes moles & 1 & $1,43 \%$ \\
\hline Laringe & 1 & $1,43 \%$ \\
\hline Melanoma de pálpebra & 1 & $1,43 \%$ \\
\hline Pâncreas & 1 & $1,43 \%$ \\
\hline Reto & $100,00 \%$ \\
\hline Rim & 1 & $1,43 \%$ \\
\hline Tireoide & 1 & $1,43 \%$ \\
\hline Útero & 1 & $1,43 \%$ \\
\hline Total & 1 & $23 \%$ \\
\hline & 1 & 2 \\
\hline
\end{tabular}

*Se uma hipótese for rejeitada quando deveria ser aceita, diz-se que foi cometido o Erro Tipo I. Se por outro lado, for aceita uma hipótese que deveria ser rejeitada, diz-se que foi cometido um Erro Tipo II. Em ambos os casos ocorreu uma decisão errada ou erro de julgamento (SIEGEL et al.,

2006).
Nesse estudo, todos os domínios no instrumento EORTC QLQC-30 apresentaram distribuição anormal de probabilidade. Porém, em decorrência dos testes terem sido feitos com $95 \%$ de confiabilidade, espera-se que $0,75 \%$ dos 15 resultados significativos talvez não sejam coerentes para afirmar melhor ou pior QV, mas sim obra do acaso (Erro Tipo I $)^{*}$, como demonstrado nos resultados da escolaridade em relação ao domínio NAV, da religião e do estado civil em relação ao domínio FAD [6].

$\mathrm{Tal}$ instrumento EORTC QLQ - $\mathrm{C}_{30}$ (version 3) possui cinco escalas funcionais: funções física, cognitiva, emocional e social e desempenho de papel; três escalas de sintomas: fadiga, dor e náusea/vômito, uma escala de saúde global/qualidade e vida; cinco outros itens que avaliam sintomas comumente relatados por doentes com câncer: 
dispneia, perda de apetite, insônia, constipação e diarreia, além de um item de avaliação de impacto financeiro do tratamento e da doença [1].

Os escores das escalas e das medidas variam de zero a 100, sendo que um valor alto do escore representa um alto nível de resposta. Assim, se o escore apresentado na escala funcional for alto, representa um nível funcional saudável, enquanto que um escore alto na escala de sintomas, representa um nível alto de sintomatologia e efeitos colaterais. Os escores dos domínios para o EORTC QLQ - C 30 (version 3 ) são calculados multiplicando-se por quatro, a média de todos os itens incluídos dentro do domínio. A avaliação dos resultados é feita mediante a atribuição de escores para cada questão, os quais podem ser transformados numa escala de zero a 100, onde zero corresponde a uma pior QV, e 100 a uma melhor QV. Porém, cada domínio é analisado separadamente [1].

Contudo, mediante a comparação das variáveis do Questionário Sociodemográfico Ocupacional (QSDO) em relação aos domínios do QLQ- C30, a Tabela 3, demonstrou melhor coerência à $\mathrm{QV}$ em indivíduos:

" Aposentado $(\mathbf{p}=0,009)$ : indivíduos aposentados em relação ao domínio DIF, tiveram (mediana $=0,00)<$ escore, indicando melhor QV nesse domínio.

" Afastado $(\mathbf{p}=\mathbf{0 , 0 0 3})$ : indivíduos que não estão afastados de suas atividades laborais, em relação ao domínio FS, tiveram (mediana $=83,30)$ > escore, indicando melhor QV nesse domínio.

" Duração da Terapêutica $(\mathbf{p}=0,019)$ : indivíduos que estão em tratamento de até cinco anos, em relação ao domínio FF, tiveram (mediana $=80,00)>$ escore, indicando melhor QV nesse domínio.

" Duração da Terapêutica $(\mathbf{p}=0,049)$ : indivíduos que estão em tratamento de até cincos anos, em relação ao domínio FE, tiveram (mediana $=100,00)>$ escore, indicando melhor QV nesse domínio.

" Duração da Terapêutica $(\mathbf{p}=0,049)$ : indivíduos que estão em tratamento de até cinco anos, em relação ao domínio FC, tiveram (mediana $=100,00)>$ escore, indicando melhor QV nesse domínio.

"Quantidade de Tratamento $(\mathbf{p}=0,002)$ : indivíduos que realizam apenas um tipo de tratamento (quimioterapia ou radioterapia), em relação ao domínio FAD, tiveram $($ mediana $=11,10)<$ escore, indicando melhor QV nesse domínio.

"Quantidade de Tratamento $(\mathbf{p}=0,020)$ : indivíduos que realizavam apenas um tipo de tratamento (quimioterapia ou radioterapia), em relação ao domínio DOR, tiveram (mediana $=0,00)<$ escore, indicando melhor QV nesse domínio.

"Idade $(\mathbf{p}=\mathbf{0 , 0 4 8})$ : indivíduos com idade até sessenta anos, em relação ao domínio DIS, tiveram (mediana = o,oo) < escore, indicando melhor QV nesse domínio.

"Renda $(\mathbf{p}=\mathbf{0}, 019)$ : indivíduos que possuem até um salário mínimo, em relação ao domínio EGS/QV, tiveram (mediana $=91,70)$ > escore, indicando melhor QV nesse domínio.

"Internações $(\mathbf{p}=\mathbf{0 , 0 4 8})$ : indivíduos que tiveram até uma internação desde o diagnóstico oncológico confirmado, em relação ao domínio FC, tiveram (mediana = 100,00) > escore, indicando melhor QV nesse domínio.

" Internações $(\mathbf{p}=\mathbf{0 , 0 3 1})$ : indivíduos que tiveram até uma internação desde o 
diagnóstico oncológico confirmado, em relação ao domínio INS, tiveram (media$\mathrm{na}=0,00)<$ escore, indicando melhor QV nesse domínio.

॥ Internações $(\mathbf{p}=\mathbf{0 , 0 0 6})$ : indivíduos que tiveram mais que uma internação desde o diagnóstico oncológico confirmado em relação ao domínio DIF, tiveram (mediana $=33,30)>$ escore, indicando melhor QV nesse domínio.

Tabela 3 - Comparação das variáveis do QSDO em relação aos domínios do EORTC QLQ - C30 dos pacientes atendidos no Hospital do Câncer - Dourados/MS, 2011.

\begin{tabular}{|c|c|c|c|c|c|}
\hline DOMÍNIO & VARIÁVEL & $\mathbf{N} \leq$ & $\mathbf{N} \geq$ & MEDIANA & $P$ \\
\hline \multicolumn{6}{|c|}{ ESCOLARIDADE } \\
\hline \multirow{2}{*}{ NAV } & Até Fundamental incompleto & 36 & 18 & 0,00 & \multirow{2}{*}{0,032} \\
\hline & Fundamental ou mais & 15 & 1 & 0,00 & \\
\hline \multicolumn{6}{|l|}{ RELIGIÃO } \\
\hline \multirow{2}{*}{ FAD } & Católico & 36 & 21 & 11,10 & \multirow{2}{*}{0,033} \\
\hline & Outros & 4 & 9 & 22,20 & \\
\hline \multicolumn{6}{|c|}{ ESTADO CIVIL } \\
\hline \multirow{2}{*}{ FAD } & Casado & 33 & 15 & 11,1 & \multirow{2}{*}{0,004} \\
\hline & Outros & 7 & 15 & 22,2 & \\
\hline \multicolumn{6}{|c|}{ APOSENTADO } \\
\hline \multirow{2}{*}{ DIF } & Não & 12 & 21 & 33,30 & \multirow{2}{*}{0,009} \\
\hline & Sim & 25 & 12 & 0,00 & \\
\hline \multicolumn{6}{|l|}{ AFASTADO } \\
\hline \multirow{2}{*}{ FS } & Não & 3 & 11 & 83,30 & \multirow{2}{*}{0,003} \\
\hline & Sim & 14 & 5 & 66,70 & \\
\hline \multicolumn{6}{|c|}{ DURAÇÃO DA TERAPÊUTICA } \\
\hline \multirow{2}{*}{ FF } & Até cinco anos & 18 & 24 & 80,00 & \multirow[b]{2}{*}{0,019} \\
\hline & Acima de cinco anos & 20 & 8 & 66,70 & \\
\hline \multirow{2}{*}{$\mathrm{FE}$} & Até cinco anos & 20 & 22 & 100,00 & \multirow{2}{*}{0,049} \\
\hline & Acima de cinco anos & 20 & 8 & 83,30 & \\
\hline \multirow{2}{*}{ FC } & Até cinco anos & 20 & 22 & 100,00 & \multirow{2}{*}{0,049} \\
\hline & Acima de cinco anos & 20 & 8 & 83,30 & \\
\hline \multicolumn{6}{|c|}{ QUANTIDADE DE TRATAMENTO } \\
\hline \multirow{2}{*}{ FAD } & Dois & 18 & 23 & 22,20 & \multirow{2}{*}{0,002} \\
\hline & Um & 22 & 5 & 11,10 & \\
\hline \multirow{2}{*}{ DOR } & Dois & 14 & 27 & 16,70 & \multirow{2}{*}{0,020} \\
\hline & $\mathrm{Um}$ & 17 & 10 & 0,00 & \\
\hline \multicolumn{6}{|l|}{ IDADE } \\
\hline \multirow{2}{*}{ DIS } & Até 60 anos & 24 & 4 & 0,00 & \\
\hline & Mais que 60 anos & 27 & 15 & 0,00 & 0,048 \\
\hline RENDA & & & & & \\
\hline & Até um s.m. & 4 & 12 & 91,70 & \\
\hline EGS/QV & Mais que um s.m. & 31 & 22 & 83,30 & 0,019 \\
\hline
\end{tabular}




\begin{tabular}{lccccc}
\hline INTERNAÇÕES & & & & & \\
\hline \multirow{2}{*}{ FC } & Até uma & 18 & 20 & 100,00 & 0,048 \\
\cline { 2 - 5 } & Mais que uma & 22 & 9 & 83,30 & 0,031 \\
\cline { 2 - 5 } INS & Até uma & 28 & 10 & 0,00 & 0,000 \\
\hline \multirow{2}{*}{ DIF } & Mais que uma & 15 & 16 & 33,30 & 0,00 \\
\hline
\end{tabular}

Notamos que os resultados caracterizaram melhor QV nos indivíduos abaixo de 60 anos em relação à DIS (um sintoma comumente referido por pacientes oncológicos) aposentados em relação à DIF, nos que não estavam afastados de suas atividades laborais em relação à FS e aos indivíduos que possuíam até um salário mínimo em relação à EGS/QV. Porém, em relação às variáveis terapêuticas, os resultados foram melhores em indivíduos que estavam em terapêutica de até 5 anos, em relação aos domínios FF, FE e FC. Já os que realizavam apenas um tipo de tratamento obtiveram melhor QV em relação aos sintomas FAD e DOR. E, por fim, os sujeitos que foram submetidos a pelo menos uma internação desde o diagnóstico confirmado, mostraram melhor resultado em relação a três escalas distintas, respectivamente, nos domínios FC, INS e DIF.

Dessa forma, os resultados demonstraram que a identificação objetiva dos domínios, quando correlacionados às variáveis sociodemográficas ocupacionais, pode favorecer o direcionamento das intervenções assistenciais que a equipe multidisciplinar oferece ao paciente on cológico em relação ao enfrentamento da doença e aos efeitos adversos do tratamento, no qual, ocasionalmente, pode comprometer a qualidade de vida dessa população.

\section{REFERÊNCIAS}

[1] BRABO, E. P. Validação para o Brasil do questionário de QV para pacientes com câncer de pulmão QLQLC 13 da organização Européia para a Pesquisa e Tratamento do Câncer. Dissertação (Mestrado em Clínica Médica) - Universidade Federal do Rio de Janeiro, Rio de Janeiro, 2006.

[2] CORREIA, F. R. Tradução, adaptação cultural e validação inicial no Brasil da Palliative Outcome Scale (POS). Dissertação (Mestrado em Enfermagem em Saúde Pública) - Escola de Enfermagem de Ribeirão Preto, Universidade de São Paulo, Ribeirão Preto, 2012.

[3] KIMURA, M.; SILVA, J. V. Índice de qualidade de vida de Ferrans e Powers. Revista de Enfermagem da USP, São Paulo, v. 43, n. esp, dez. 2009. Disponível em: <http://www.scielo.br/scielo.php?script=sci_arttext\&pid=Soo8o$-62342009000500014 \& \operatorname{lng}=e n \& n r m=i s o>$. Acesso em 01 nov. 2013. http:// dx.doi.org/10.1590/Soo80-62342009000500014

[4] MACHADO, S. M.; SAWADA, N. O. Avaliação da qualidade de vida de pacientes oncológicos em tratamento quimioterápico adjuvante. Texto Contexto de Enfermagem, Florianópolis, v. 17, n. 4, out/dez., 2008. Disponível em: 
<http://www.scielo.br/scielo.php?script=sci_arttext\&pid=So104$-07072008000400017 \& \operatorname{lng}=$ en\&nrm=iso $>$. Acesso em: o1 Nov. 2013. http:// dx.doi.org/10.1590/So104-07072008000400017.

[5] NUCCI, N. A. G.. Qualidade de vida e câncer: um estudo compreensivo. 2003. Tese (Doutorado em Psicologia) - Faculdade de Filosofia, Ciências e Letras de Ribeirão Preto, Universidade de São Paulo, Ribeirão Preto, 2003. Disponível em: <http://www.teses.usp.br/teses/disponiveis/59/59137/tde-27012004-222429/>. Acesso em: 11 mar. 2012.

[6] SIEGEL, S. et al. Estatística não-paramétrica para ciências do comportamento. Porto Alegre: Artmed, 2006.

CINTIA RACHEL GOMES SALES fisioterapeuta, enfermeira, mestre em Psicologia da Saúde da Universidade Católica Dom Bosco (UCDC). Docente do Centro Universitário da Grande Dourados (UNIGRAN) - e-mail:ftcintia@hotmail.com

JOSÉ CARLOS ROSA PIRES médico psiquiatra, doutor em Ciências Médicas da UNICAMP e pós-doutor da Universidade de Lisboa. Docente da Universidade Católica Dom Bosco

IDALINA CRISTINA FERRARI JÚLIO enfermeira e especialista em Oncologia. Docente da Universidade Estadual de Mato Grosso do Sul 